\title{
A radiomics model to predict the invasiveness of thymic epithelial tumors based on contrast-enhanced computed tomography
}

\author{
XIANGMENG CHEN $^{1,2^{*}}$, BAO FENG $^{1,3^{*}},{\text { CHANGLIN } \mathrm{LI}^{3}, \text { XIAOBEI DUAN }}^{4}$, YEHANG CHEN $^{3}$, \\ ZHI LI ${ }^{3}$, ZHUANGSHENG LIU ${ }^{1}$, CHAOTONG ZHANG ${ }^{1}$ and WANSHENG LONG ${ }^{1}$ \\ ${ }^{1}$ Department of Radiology, Jiangmen Central Hospital, Jiangmen, Guangdong 529030; \\ ${ }^{2}$ Department of Medical Imaging Center, The First Affiliated Hospital of Jinan University, Guangzhou, Guangdong 510632; \\ ${ }^{3}$ School of Electronic Information and Automation, Guilin University of Aerospace Technology, Guilin, Guangxi 541004; \\ ${ }^{4}$ Department of Nuclear Medicine, Jiangmen Central Hospital, Jiangmen, Guangdong 529030, P.R. China
}

Received July 25, 2019; Accepted January 16, 2020

DOI: $10.3892 /$ or.2020.7497

\begin{abstract}
In the present study, we aimed to construct a radiomics model using contrast-enhanced computed tomography (CT) to predict the pathological invasiveness of thymic epithelial tumors (TETs). We retrospectively reviewed the records of 179 consecutive patients ( 89 females) with histologically confirmed TETs from two hospitals. The 82 low- and 97 high-risk TETs were assigned to training (90 tumors), internal validation (49 tumors) and external validation (40 tumors) cohorts. Radiomics features extracted from preoperative contrast-enhanced chest CT were selected using least absolute shrinkage and selection operator logistic regression. Three prediction models were developed using multivariate logistic regression analysis. Their performance and clinical utility were assessed using receiver operating characteristic curves and the DeLong test, respectively. Eight radiomics features with non-zero coefficients were used to develop a radiomics score, which significantly differed between low- and high-risk TETs $(\mathrm{P}<0.001)$. The subjective finding, infiltration, was independently associated with high-risk TETs. Prediction models based on infiltration alone, the radiomics signature alone, and both these parameters showed diagnostic accuracies of $72.2 \%$ [area under curve (AUC), 0.731; 95\% confidence interval (CI): 0.627-0.819; sensitivity, $85.7 \%$; specificity, 60.4\%], 88.9\% (AUC, 0.944; 95\% CI: 0.874-0.981; sensitivity, $92.9 \%$; specificity, $85.4 \%$ ), and $90.0 \%$ (AUC, 0.953; 95\% CI: 0.887-0.987; sensitivity, 92.9\%; specificity, $87.5 \%$ ), respectively. Decision-curve analysis showed that the combined model added more net benefit than the single-parameter models. In conclusion, a radiomics signature based on contrast-enhanced $\mathrm{CT}$ has the potential to differentiate
\end{abstract}

Correspondence to: $\mathrm{Dr}$ Wansheng Long, Department of Radiology, Jiangmen Central Hospital, 23 North Road, Pengjiang Zone, Jiangmen, Guangdong 529030, P.R. China

E-mail: jmlws2@163.com

Key words: radiomics, computed tomography, thymic epithelial tumor, tumor invasiveness, quantitative imaging between low- and high-risk TETs. The model incorporating the radiomics signature and subjective finding may facilitate the individualized, preoperative prediction of the pathological invasiveness of TETs.

\section{Introduction}

Almost half of all anterior mediastinal tumors (47\%) are thymic epithelial tumors (TETs) $(1,2)$. The 2014 consensus statement of the International Thymic Malignancy Interest Group on the histological classification of TETs informed the 2015 update of the World Health Organization (WHO) TET classification. According to this WHO classification, TETs can be subdivided into thymoma (subtypes A, AB, B1, B2 and B3) and thymic carcinoma (subtype $\mathrm{C}$ ), in the order of increasing degree of malignancy $(3,4)$. In patients who have TETs with a low risk of malignancy (subtypes A, AB and B1), complete surgical resection without adjuvant or neoadjuvant chemotherapy is typically sufficient. In contrast, patients who have TETs with a high risk of malignancy (subtypes B2, B3 and C) require a combination of treatments, including chemotherapy, radiotherapy, and/or surgical resection $(5,6)$. Pretreatment needle biopsy is a reliable method of diagnosing TETs. However, a small biopsy specimen may not always be representative of the entire tumor, and deep biopsy is an invasive procedure that poses the risk of complications $(2,7)$.

Different imaging modalities have been used for the preoperative assessment of TETs. The most common of these is thoracic computed tomography (CT) with intravenous contrast enhancement. On enhanced CT images, irregular contours, heterogeneous enhancement, and mediastinal fat invasion strongly suggest a high-risk TET (8-10). However, the value of qualitative CT features in determining the degree of tumor invasiveness remains controversial. Furthermore, the assessment of qualitative imaging features is subjective and subject to inter-reader variability. Therefore, an effective and objective approach for preoperative determination of the subtype of TET is urgently required.

Radiomics is a field of medical research in which a large amount of high-quality, quantitative, mineable data is extracted from conventional medical images $(11,12)$. The 
application of radiomics techniques to further the development of personalized medicine in the field of oncology has already provided insights on the detection and classification of tumors, and the assessment of therapeutic response (13). Objective and quantitative radiomics signatures that may serve as prognostic biomarkers have already been developed for tumors such as brain glioblastoma, breast cancer and lung cancer (13-15). However, to date, only one study (16) has investigated the relationship between CT-based radiomics parameters and the histological classification of TETs. Furthermore, that study included only 16 patients and did not involve a comprehensive quantitative texture analysis to reflect whole-tumor radiomics characteristics.

Therefore, the purpose of our research was to develop and validate a radiomics model that incorporated both the radiomics signature and subjective CT features for individual preoperative prediction of the pathological invasiveness of TETs.

\section{Materials and methods}

Patient selection. The study protocol was approved by the Institutional Review Boards of Jiangmen Central Hospital and The Fifth Affiliated Hospital of Sun Yat-sen University. The need for informed consent was waived as our study has a retrospective design. We searched the electronic medical database of the two hospitals for the records of all patients who underwent resection for thymic neoplasms and histopathologic diagnosis between February 2009 and March 2019. The following inclusion criteria were applied: i) histopathologically confirmed primary TETs; ii) CT images available in the picture archiving and communication system; iii) thoracic spiral CT scan with intravenous contrast dual-phase enhancement was performed within 4 weeks before surgery; iv) no history of prior resection for thymic neoplasm or other malignant tumor, and v) no history of biopsy, chemotherapy, or radiotherapy prior to the primary thoracic CT scans.

After the application of the selection criteria, we enrolled 139 patients from center 1, including 69 men and 70 women. The median age of the patients was 54 years (age range, 19-81 years). Between 25 February 2009 and 2 December 2016, 90 patients (42 men, 48 women; median age, 53 years; age range, 19-81 years) were identified, and these patients comprised the training cohort. Between 15 December 2016 and 10 October 2018, 49 consecutive patients (27 men, 22 women; median age, 55 years; age range, 26-75 years) were selected using the same criteria as those used for the training cohort; these patients formed an internal validation cohort. In center 2, 40 consecutive patients ( 21 men, 19 women; median age, 57 years; age range, 28-83 years) were enrolled between March 2014 and March 2019 using the same criteria as those used in center 1 , and constituted the external validation cohort. A flowchart of the patient-selection process is shown in Fig. 1.

Thoracic contrast-enhanced CT scan protocol. Chest CT was performed using four different CT scanners: Aquilion One-64 (Toshiba Medical Systems), Somatom Sensation-16 and Dual-energy Force (Siemens Medical Solutions), and Discovery HD 750 (GE Medical Systems). The scanning parameters were as follows: $120 \mathrm{kVp}$; 80-200 mAs; rotation time, $0.5 \mathrm{sec}$; field of view, $350 \times 350 \mathrm{~mm}$; detector collimation,
$64 \times 0.625 \mathrm{~mm}$ or $16 \times 0.6 \mathrm{~mm}$; and matrix, $512 \times 512$. Two different slice thicknesses of $3.0 \mathrm{~mm}(\mathrm{n}=164)$ and $2.5 \mathrm{~mm}$ $(n=15)$ were obtained, and the corresponding images were reconstructed using soft-tissue and lung kernels, respectively.

CT scanning was conducted during a single deep breath-hold, with the patient in a supine position. The scanning field extended from the level of the thoracic inlet to the level of the adrenal glands. First, a conventional plain CT scan was obtained, and then, an iodinated contrast agent was injected into the antecubital vein of the patient (Ultravist, Bayer Schering Pharma; dose, $1.5 \mathrm{ml} / \mathrm{kg}$; injection rate, $3.5-4.5 \mathrm{ml} / \mathrm{sec}$ ). Enhanced CT scans in the arterial and venous phases were obtained at 30 and $60 \mathrm{sec}$, respectively, after the injection of the contrast material.

Analysis of subjective CT findings. All CT scans were independently reviewed by two radiologists, one with 10 years of experience (Reader 1) and the other with 25 years of experience (Reader 2) in thoracic radiology. Both radiologists were blinded to the clinical history and final histopathological diagnosis. For lesion evaluation, they reviewed images obtained using both the mediastinal [level, $30 \mathrm{HU}$ (hounsfield unit); width, $350 \mathrm{HU}$ ] and lung (level, -600 HU; width, $1500 \mathrm{HU}$ ) window settings.

Subjective CT findings were evaluated as follows: i) location (left, right, or midline in the anterior mediastinum); ii) diameter (average of the maximum long axis and the maximum short axis perpendicular to the long axis in the same transverse cross-sectional slice); iii) margin (regular or irregular), iv) calcification (absent or present); v) enhancement pattern (homogeneous or heterogeneous); vi) enhancement degree (none, minimal, moderate, or severe), and vii) infiltration (absent or present). Minimal, moderate, and severe enhancement was defined as enhancement less than, equal to, and greater than that of the chest-wall muscle, respectively (17). Infiltration was defined as the disappearance of the fat-density plane between the tumor and the adjacent tissue (18). Consensus was reached through discussion.

Inter-reader agreement and subjective findings model. Interobserver agreement for the evaluation of the subjective findings was determined using the Cohen kappa test as follows: $\kappa<0.00$, poor agreement; $\kappa=0.00-0.20$, slight agreement; $\kappa=0.21-0.40$, fair agreement; $\kappa=0.41-0.60$, moderate agreement; $\kappa=0.61-0.80$, substantial agreement; and $\kappa=0.81-1.00$, almost perfect agreement (19).

Age, sex, and subjective CT findings (lesion location, diameter, margin, calcification, enhancement pattern, enhancement degree, and infiltration) were compared between study groups by using the t-test, Chi-square test, or Mann-Whitney U test, as required. Variables that were found to be significant on univariate analysis were entered into multivariate logistic regression analysis. The results were used to construct a model of the subjective findings.

Pathological diagnosis. The median time between CT imaging and surgery was 18 days. Complete resection was achieved in $159(88.8 \%)$ patients and incomplete resection was achieved in $20(11.2 \%)$ patients. TET specimens were fixed with formalin and stained with hematoxylin and eosin. Pathological analysis 
was performed by two pathologists, one with 10 years of experience and the other with 15 years of experience in thoracic pathological analysis. Both pathologists were blinded to the clinical history and chest CT findings. TETs were evaluated and classified according to the epithelial-tumor-cell morphology, relative proportion of the nontumoral lymphocytic component, immunohistochemical findings, and degree of resemblance to the normal thymic structure. The tumors were subdivided into a low-risk group (subtypes $\mathrm{A}, \mathrm{AB}$ and $\mathrm{B} 1$ ) and a high-risk group (subtypes B2, B3 and C) according to the $2015 \mathrm{WHO}$ histological classification $(3,4)$. All tumors were also staged according to the Masaoka-Koga clinical-pathologic staging system (2).

Volume of interest segmentation. Volume of interest (VOI) segmentations were manually implemented by the same two readers who assessed the subjective $\mathrm{CT}$ findings. Using our in-house tool developed on MATLAB 2016 (Mathworks), the TET lesions were manually delineated on venous-phase axial CT images because most TETs exhibit significant enhancement and relatively clear margins in this phase. Contouring was performed slightly within the tumor borders in order to avoid the adjacent tissues, such as mediastinal fat, tracheal, vessel, and lung tissues. For lesions that were ambiguous in the axial plane, the corresponding sagittal and coronal planes were referenced. Whole tumor volume was contoured on two-dimensional images. For each lesion, two VOIs were obtained. Grayscale discretization and isotropic resampling were used to reconstruct the VOIs (Fig. 2).

Radiomics feature extraction and radiomics signature model. We extracted 10,394 three-dimensional radiomics features that were based on factors such as shape, intensity, texture, and wavelets. Feature extraction was performed using our in-house software developed with MATLAB 2016 (Mathworks). We randomly selected 20 patients in the training cohort, and Readers 1 and 2 performed tumor segmentations for these patients. Reader 1 repeated this same procedure 1 week later. Intraclass coefficients (ICCs) were calculated to assess the interobserver and intraobserver reproducibility of the extracted radiomics features. The remaining TET lesions were segmented by Reader 1 . Radiomics features were extracted from all segmentations. The value of each feature in distinguishing between high- and low-risk TETs was determined using the Mann-Whitney U test. Features that significantly differed between the low- and high-risk TETs and had an intraclass coefficient (ICC) $>0.75$ were filtered using Pearson correlation coefficient analysis and subjected to least absolute shrinkage and selection operator (LASSO) logistic regression with a 10 -fold cross validation (20).

Radiomics features that were found to have non-zero coefficients on LASSO logistic regression were subjected to Pearson correlation analysis. Features with Pearson correlation coefficients of $<0.9$ were selected as independent factors for building a radiomics-signature model. We created the radiomics signature via the linear combination of the chosen radiomics features. For each patient, a radiomics score was calculated using the linear combination of the chosen features, weighted according to their coefficients (Appendix 1 and Table S1). The radiomics signature was employed to construct a model for the detection of high-risk TETs in the training cohort.
179 Patients with pathologically confirmed thymic epithelial tumors who received preoperative thoracic contrast-enhanced CT examination

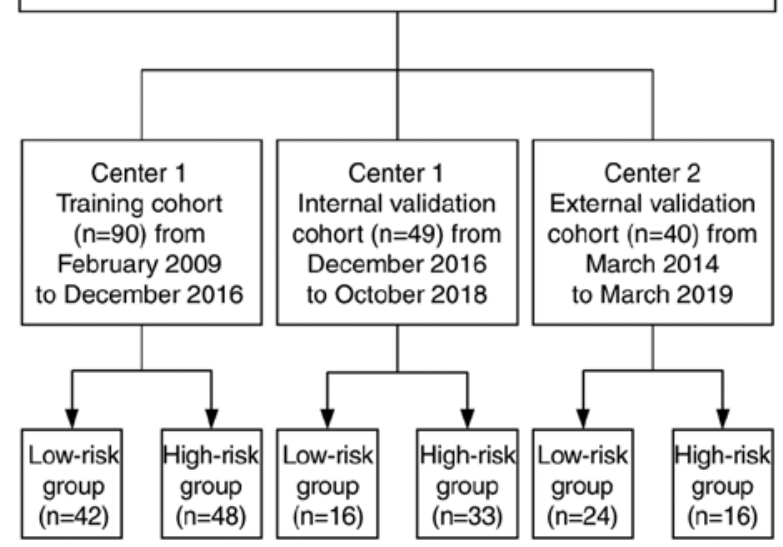

Figure 1. Flowchart of the patient-selection process. CT, computed tomography.

Combined model. Multivariate logistic regression analysis was performed to construct a model including both relevant subjective findings and the radiomics signature. Backward step-wise elimination was used with the likelihood ratio test and Akaike information criterion.

Model validation, performance, and potential clinical value. Receiver operating characteristic (ROC) curve analysis was used to evaluate the performances of all three models. We calculated the sensitivity, specificity, positive and negative predictive values (PPV and NPV, respectively), accuracy, and area under the curve (AUC). Next, cutoff values were chosen such that the sum of the sensitivity value and specificity value was maximized. Finally, we compared the AUCs of the three models by using the DeLong test. The calibrations of the subjective finding, radiomics signature, and combined models were assessed using calibration curves and the Hosmer-Lemeshow test. Moreover, stratified analyses of clinical variables (sex, age, CT device, and CT slice thickness) were carried out to assess the impact of the combined model in the training, internal and external validation cohorts.

To estimate the clinical usefulness of the three models, we quantified their net benefits for multiple threshold probabilities by using decision-curve analysis (DCA) (21).

Statistical analysis. All statistical analyses were performed using R software (v3.0.1; http://www.rproject.org) and MATLAB. LASSO analysis, ROC curve analysis,Pearson correlation coefficient analysis, and DCA were performed using the glmnet (https://cran.r-project.org/web/packages/glmnet/ index.html), pROC (https://www.rdocumentation.org/packages/ pROC/versions/1.12.1),corfunction(https://www.rdocumentation. org/packages/stats/versions/3.4.1/topics/cor) and dca.r (https://www.rdocumentation.org/packages/DecisionCurve/ versions/1.4) packages, respectively. Parametric differences between the low- and high-risk groups were compared using the two-tailed t-test. $\mathrm{P}<0.05$ was considered to indicate a statistically significant difference. 

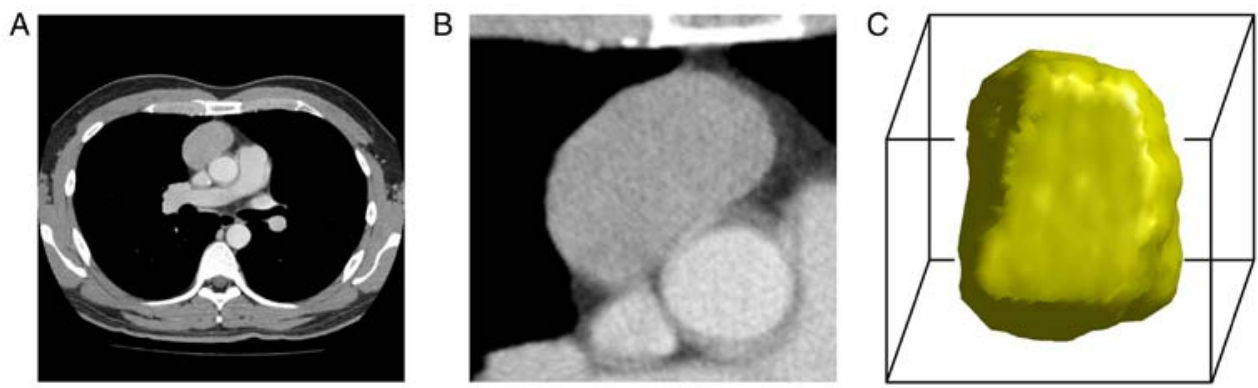

Figure 2. Volume of interest segmentation of a thymic epithelial tumor. (A) One regular soft-tissue mass with moderate and homogeneous enhancement is identified in the anterior mediastinum on a venous-phase computed tomography scan. (B) Manual segmentation of the lesion on the same transverse slice. (C) Three-dimensional volumetric reconstruction of the lesion obtained using the MATLAB platform.

\section{Results}

General patient characteristics. In the two centers, there were 82 patients in the low-risk TET group (type A, 20 patients; type $A B, 41$ patients; and type B1, 21 patients), and 97 patients in the high-risk TET group (type B2, 57 patients; type B3, 22 patients; and type C, 18 patients). We found no significant differences between the low-risk and high-risk groups in terms of sex (training cohort, $\mathrm{P}=0.799$; internal validation cohort, $\mathrm{P}=0.617$; external validation cohort, $\mathrm{P}=0.796$ ) and age (training cohort, $\mathrm{P}=0.898$; internal validation cohort, $\mathrm{P}=0.794$; external validation cohort, $\mathrm{P}=0.744)$. The locations of the TET lesions were as follows: Left anterior mediastinum, 52 lesions; right anterior mediastinum, 69 lesions; and midline anterior mediastinum, 58 lesions (Table I). Patient distribution according to Masaoka-Koga stage were classified as follows: Stage I, 76 patients; stage II, 56 patients; stage III, 30 patients; stage IV, 17 patients.

Subjective-finding model. In the training cohort, the mean tumor diameter did not significantly differ between the low-risk $(4.78 \pm 2.17 \mathrm{~cm})$ and high-risk tumors $(4.77 \pm 2.04 \mathrm{~cm}$; $\mathrm{P}=0.926)$. Irregular margins were significantly more frequent in high-risk tumors $(n=25)$ than in low-risk tumors $(n=11$; $\mathrm{P}=0.012$ ). Calcifications were slightly less common in low-risk tumors $(n=12)$ than in high-risk tumors $(n=16)$, although the difference was not significant $(\mathrm{P}=0.626)$. Additionally, there were no significant differences in the pattern $(\mathrm{P}=0.181)$ and degree of enhancement $(\mathrm{P}=0.491)$ on venous-phase $\mathrm{CT}$ images between the two study groups. Infiltration was significantly less frequent in low-risk tumors $(n=6)$ than in high-risk tumors $(\mathrm{n}=29 ; \mathrm{P}<0.001)$. These findings in the internal and external validation cohorts were similar. Patient characteristics in the training and two validation cohorts are presented in Table I.

Using multivariate logistic regression, we identified infiltration [odds ratio (OR), 0.109; 95\% confidence interval (CI): 0.039-0.309; $\mathrm{P}<0.001]$ as an independent predictor in the subjective findings model. The interobserver agreement for various qualitative subjective $\mathrm{CT}$ features of TETs ranged from $\kappa=0.557$ to $\kappa=0.972$. Calcification showed perfect agreement $(\kappa=0.944,95 \%$ CI: 0.879-0.972), while enhancement pattern $(\kappa=0.798,95 \%$ CI: $0.692-0.876)$ and enhancement degree ( $\kappa=0.728,95 \%$ CI: 0.623-0.830) showed substantial agreement. Lesion margins ( $\kappa=0.656,95 \% \mathrm{CI}: 0.557-0.786)$ and infiltration $(\kappa=0.670,95 \%$ CI: $0.560-0.738)$ showed moderate agreement.
Radiomics feature selection and radiomics signature. Of the 10,394 radiomics features, 294 had ICC values between 0.75 and 1.0 and showed significant differences between the low-risk and high-risk groups $\left(\mathrm{P}=1.112 \times 10^{-4}-0.049\right)$ in the training cohort. These 294 features were subjected to LASSO logistic regression, which identified 8 features with non-zero coefficients. No repeatability was shown between these 8 radiomics features through the Person correlation coefficient analysis (Table SII). These 8 features were used to create a formula for the calculation of a radiomics score (Table II; Fig. 3). The radiomics score thus calculated significantly differed between the low-risk and high-risk tumors in the training $(\mathrm{P}<0.001)$, internal validation $(\mathrm{P}<0.001)$ and external validation cohorts $(\mathrm{P}=0.009)$ (Table I; Fig. 4).

Among the 8 selected features, the shape-related features eccentricity and solidity had smaller values for high-risk tumors than for low-risk tumors. The texture-related features small zone emphasis_gray level size zone matrix (SZE_GLSZM), small zone high gray-level emphasis_gray level size zone matrix (SZHGE_GLSZM) and high gray-level run emphasis_graylevel run-length matrix (HGRE_GLRLM) also had smaller values for the high-risk tumors than for the low-risk tumors. Variance of the gray-level co-occurrence matrix (GLCM) under low gray-scale ranges, a texture spatial distribution-related feature, had larger values for low-risk tumors than for high-risk tumors (Table II).

Model construction and performance. Multivariate analysis revealed that infiltration (OR, 0.208; 95\% CI: 0.047-0.930; $\mathrm{P}=0.040)$ and the radiomics signature (OR, 7.444; 95\% CI: 2.981-18.585; $\mathrm{P}<0.001)$ were independent predictors of high-risk TETs (Table III). Therefore, we developed three models: One based on infiltration alone (subjective finding model), one based on the radiomics signature alone (radiomics signature model), and one based on the combination of these two factors (combined model).

In the training cohort, sensitivity, specificity, accuracy, PPV and NPV were lower in the subjective finding model than in the radiomics signature and combined models. The cut-off values for the subjective finding model, radiomics signature model, and combined model were $0.1710,-0.3534$ and 0.3923 , respectively. The AUC value of the subjective finding model $(0.731,95 \%$ CI: 0.627-0.819) was significantly lower than the AUC values of the radiomics signature model $(0.944,95 \%$ CI: 0.874-0.981; $\mathrm{P}<0.001)$ and the combined model $(0.953,95 \%$ CI, 0.887-0.987; $\mathrm{P}<0.001)$. However, no significant difference was found between 


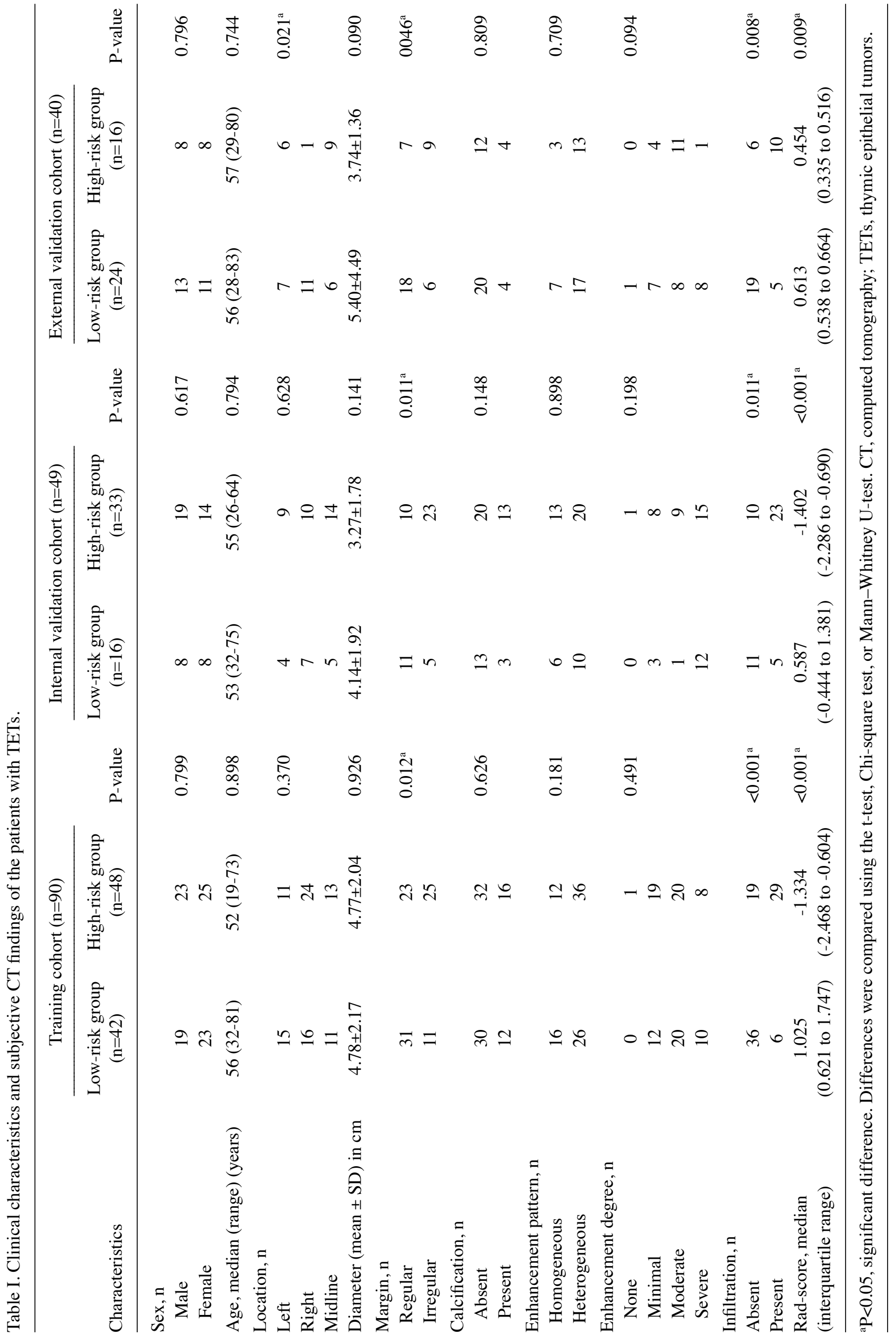


A $\quad 695959555041352217139854322$

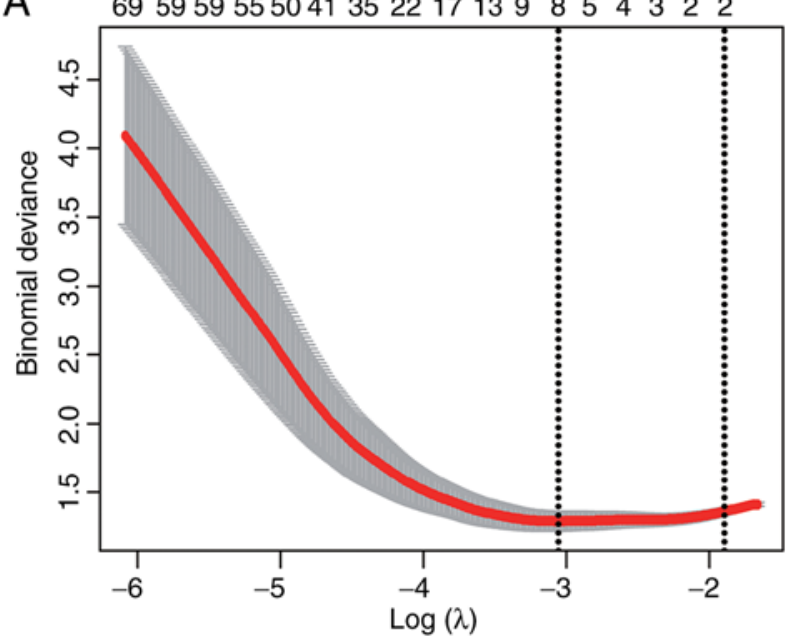

B

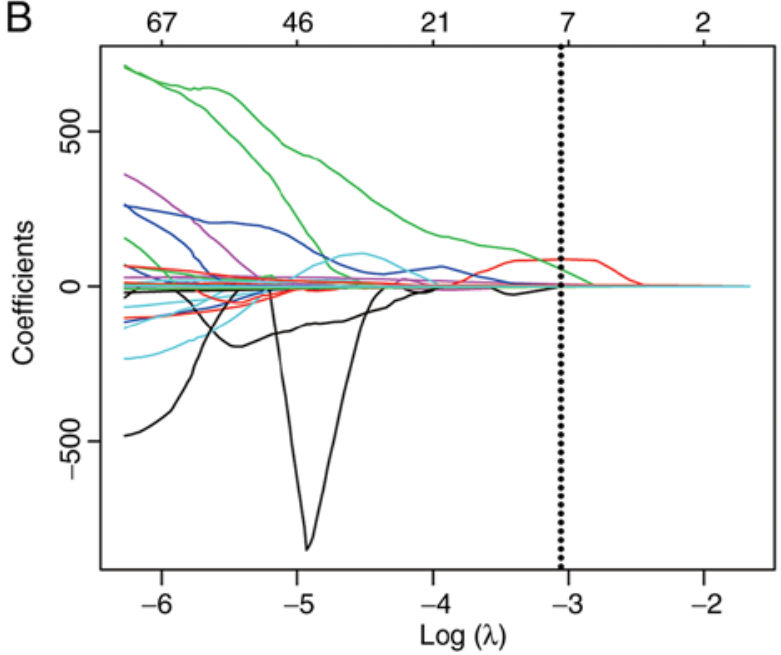

Figure 3. Radiomics-feature selection. (A) The tuning parameter $(\lambda)$ was selected using 10 -fold cross-validation based on minimum criteria. Log $(\lambda)$ is plotted on the $\mathrm{x}$-axis, and binomial deviance is plotted on the $\mathrm{y}$-axis. The dotted vertical lines indicate optimal values determined using the minimum criteria and one standard error of the minimum criteria (1-SE). Optimal $\lambda=0.04804 ; \log (\lambda)=-3.0357$. (B) LASSO coefficient profiles of whole features. Coefficient profiles are plotted against $\log (\lambda)$. The vertical line is drawn where the eight optimal radiomics features with non-zero coefficients were indicated in the plot according to 10-fold cross-validation.
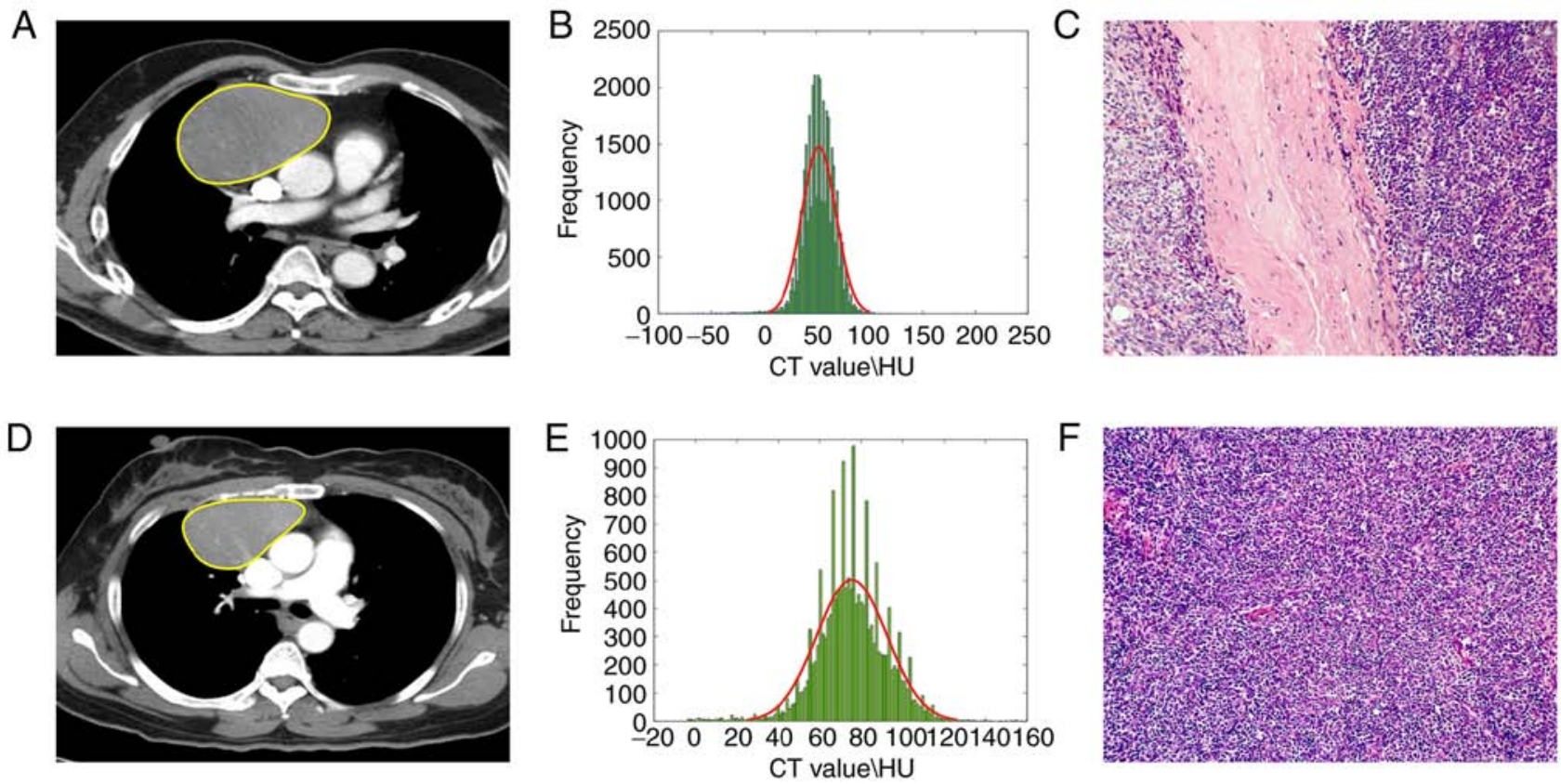

Figure 4. Low-risk and high-risk TETs. (A) Transverse contrast-enhanced CT shows a homogeneous, enhanced soft-tissue mass measuring 8.3-cm in the anterior mediastinum in a 56-year-old man. (B) Histogram analysis of the entire lesion shows a low cumulative HU value but high relative frequency. The horizontal axis shows the CT-value distribution, and the vertical axis represents the numbers in the mass. The radiomics score was 1.401. (C) This mass is confirmed as a low-risk TET (WHO grade AB) on pathological analysis (hematoxylin and eosin, magnification x200). (D) Transverse contrast-enhanced CT shows a homogeneous, enhanced soft-tissue mass measuring 6.5-cm in the anterior mediastinum in a 48-year-old woman. (E) Histogram analysis of the entire lesion shows high cumulative HU value but low relative frequency. The horizontal axis shows the CT-value distribution, and the vertical axis represents the numbers in the mass. The radiomics score was-0.409. (F) This mass is confirmed as a high-risk TET (WHO grade B2) on pathological analysis (hematoxylin and eosin, magnification x200). CT, computed tomography; HU, hounsfield unit; TETs, thymic epithelial tumors.

the AUCs of the radiomics signature and combined models $(\mathrm{P}=0.266)$. The diagnostic performance of each model and their ROC curves were summarized in Table IV and Fig. 5. The calibration curves for each model demonstrated good agreement. The Hosmer-Lemeshow tests were not significant (all P>0.05), representing a good fit (Table SIII, Fig. S1). As shown in Fig. S2, the stratified analysis (Appendix 2) showed that the performance of the combined model was not affected by sex, age, CT device, or CT slice thickness (Delong test; $\mathrm{P}>0.05$ ).

Clinical value of the models. DCA showed that the net benefit of the combined model (threshold probability, 0.01-0.87) was greater than those of the treat-all-patients and treat-no-patients schemes. Furthermore, the net benefit of the combined 
Table II. Eight radiomics features with non-zero LASSO coefficients in the training cohort.

\begin{tabular}{lccr}
\hline Characteristics & Low-risk group $(\mathrm{n}=42)$ & High-risk group $(\mathrm{n}=48)$ & P-value \\
\hline GLSZM_SZE_0.5_1.5_Lloyd_32 & $0.676 \pm 0.040$ & $0.659 \pm 0.024$ & $0.048^{\mathrm{a}}$ \\
GLSZM_SZHGE_0.5_2_Lloyd_8 & $9.010 \pm 1.726$ & $8.061 \pm 1.906$ & $0.010^{\mathrm{a}}$ \\
GLCM_Variance_1_1.2_Equal_16 & $0.077 \pm 0.002$ & $0.076 \pm 0.002$ & $0.019^{\mathrm{a}}$ \\
GLCM_Variance_1_2_Lloyd_32 & $0.018 \pm 0.011$ & $0.014 \pm 0.006$ & $0.032^{\mathrm{a}}$ \\
GLCM_Variance1.5_1.2_Equal_16 & $0.077 \pm 0.002$ & $0.077 \pm 0.002$ & $0.026^{\mathrm{a}}$ \\
GLRLM_HGRE_1.5_0.67_Lloyd_8 & $25.550 \pm 2.914$ & $23.369 \pm 2.443$ & $<0.001^{\mathrm{a}}$ \\
Eccentricity & $0.716 \pm 0.092$ & $0.666 \pm 0.099$ & $0.008^{\mathrm{a}}$ \\
Solidity & $0.795 \pm 0.109$ & $0.707 \pm 0.105$ & $<0.001^{\mathrm{a}}$ \\
\hline
\end{tabular}

${ }^{\mathrm{a}} \mathrm{P}<0.05$, significant difference. All P-values were calculated using the Mann-Whitney U test. LASSO, least absolute shrinkage and selection operator. GLSZM, gray-level size zone matrix; SZE, small zone emphasis; SZHGE, small zone high gray-level emphasis; GLCM, gray-level co-occurence matrix; GLRLM, gray-level run-length matrix; HGRE, high gray-level run emphasis.

Table III. Multivariate logistic regression analysis of parameters for distinguishing between low-risk and high-risk TETs.

\begin{tabular}{lccc}
\hline Intercept and variable & $\beta$ & Odds ratio $(95 \% \mathrm{CI})$ & P-value \\
\hline Intercept & 0.573 & & 0.212 \\
Infiltration & -1.570 & $0.208(0.047-0.930)$ & $0.040^{\mathrm{a}}$ \\
Radiomics signature & 2.007 & $7.444(2.981-18.585)$ & $<0.001^{\mathrm{a}}$ \\
\hline
\end{tabular}

${ }^{\text {a }}<<0.05$, significant difference. $\beta$, regression coefficient; $C I$, confidence interval; TETs, thymic epithelial tumors.

model was greater than that of the subjective finding model (0.01-0.42) and similar to that of the radiomics signature model (0.01-0.84; Fig. 6).

\section{Discussion}

The preoperative prediction of the WHO histological subtype of TETs may help determine whether surgical tumor resection alone is sufficient or if preoperative adjuvant treatment is required. In the present study, we investigated the ability of enhanced CT-based radiomics analysis to predict the risk status of patients with TETs. A radiomics model combining subjective findings and a radiomics signature was established, and showed better performance in the training and two validation cohorts than the prediction model based on subjective findings. Thus, radiomics features could serve as a noninvasive method to preoperatively predict the risk of malignancy, which has implications for treatment decisions.

Studies have shown that several CT features may be helpful in differentiating the invasiveness of TET subtypes. Tomiyama et al, found that tumor size did not significantly differ among the various TET subtypes (22). Another study revealed that homogeneous enhancement tended to indicate a low risk of malignancy, while heterogeneous enhancement indicated a high risk of malignancy (17). However, these density findings were not observed in our study. Sadohara et al, reported that tumors with smooth contours likely carried a low risk of malignancy (23). Infiltration, which reflects invasion of the adjacent structures, suggests a high risk of malignancy (24). In the present study, lesion margins and infiltration significantly differed between the two TET subtypes in both the training and validation cohorts, which is consistent with previous findings $(9,23,24)$. The major drawbacks of subjective radiological evaluation were inter-reader variability and weak repeatability. Only moderate interobserver agreement was found for the characterization of margins and infiltration $(\kappa=0.656$ and 0.670 , respectively), even though two experienced thoracic radiologists performed the assessments in our study. Thus, the current descriptive criteria need to be replaced with more objective and quantitative criteria.

Our combined model was constructed in a training cohort of 90 patients (AUC, 0.953 ) and was confirmed to have a good predictive performance in two validation cohorts (internal validation, 49 patients, AUC: 0.852; external validation, 40 patients, AUC: 0.826). Two shape-related features, eccentricity and solidity, were selected for the prediction of TET-risk status. Eccentricity was a first-order shape feature obtained by calculating the long axis-to-short axis ratio within the tumor volume. This feature describes the image compactness, and its value is 1 when the volume is close to a sphere. The greater eccentricity in low-risk tumors than in high-risk tumors could be interpreted as reflecting the expansive growth pattern and slow tumor-doubling time in the former group $(25,26)$. Solidity is also a first-order shape feature that represents image compactness. Solidity was determined by calculating the number of voxels in the convex hull of the VOI. This feature also had higher values in low-risk tumors than in high-risk tumors. This finding could be explained by low-risk TETs 
A

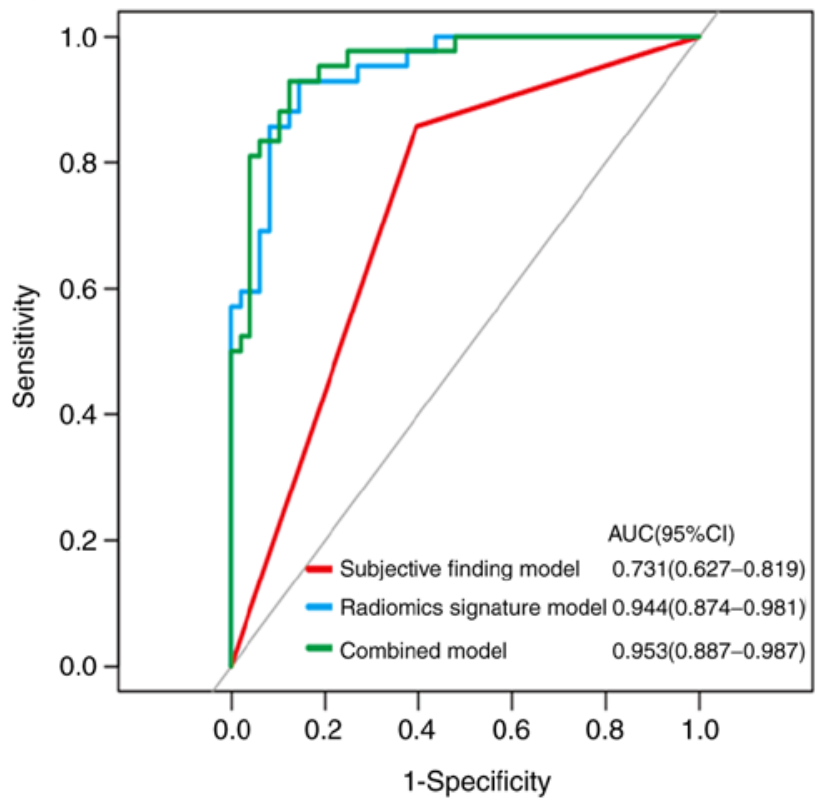

B

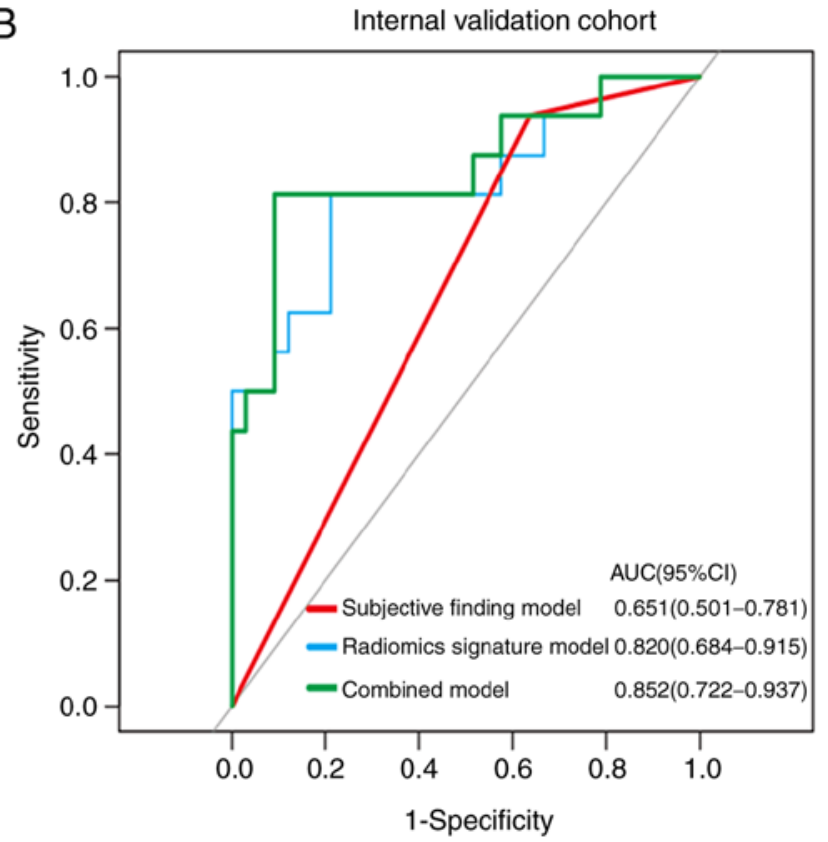

C External validation cohort

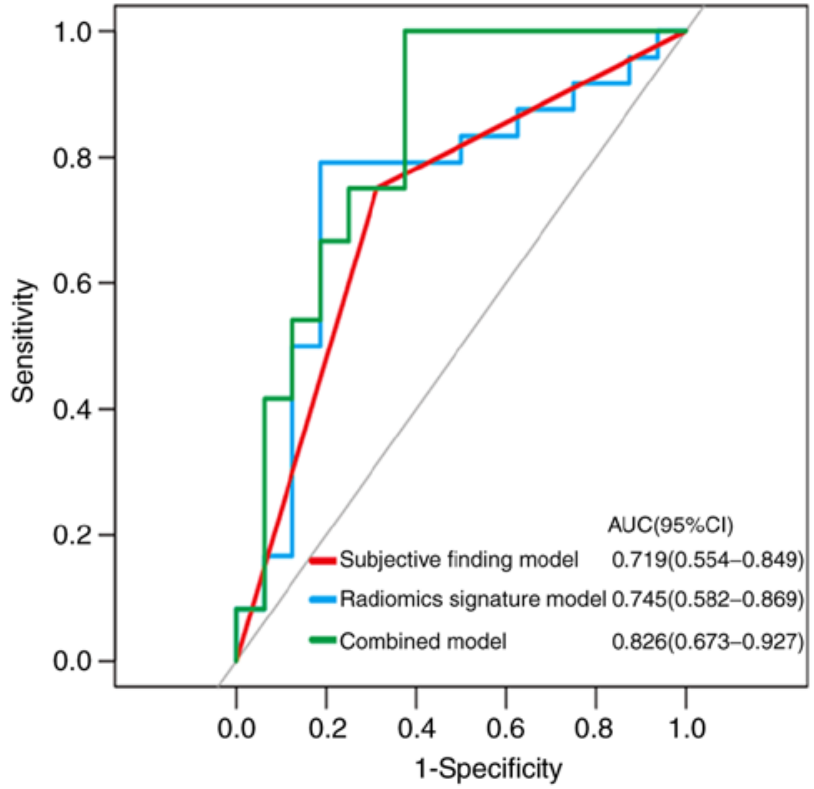

Figure 5. Receiving operating characteristic (ROC) curves of the subjective finding model (red), radiomics signature model (blue), and combined model (green) in the training (A), internal (B) and external validation (C) cohorts. AUC, area under the curve; CI, confidence interval.

being mostly well-differentiated with a complete capsule and high-risk TETs being typically poorly differentiated without a capsule $(27,28)$. These two quantitative radiomics parameters are based on 3D segmentation and offer an advantage over viewing the lesion in a single plane. Moreover, these features may distinguish and characterize the morphological measures of the edge characteristics of TETs more reproducibly and accurately than visual assessment.

Textural radiomics parameters reflect the intra-tumor heterogeneity and cannot be identified visually. In our study, the variance of the GLCM under the low gray-scale range was valuable for predicting the invasiveness of TETs. This variance was greater in low-risk tumors than in high-risk tumors, probably because more voxels with regular signal intensity spatial distribution were required to represent low-grade, highly differentiated tumor cells. The texture unit-related features SZE_GLSZM, SZHGE_GLSZM, and HGRE_GLRLM had lower values in high-risk tumors than in low-risk tumors, probably due to greater tumor-cell accumulation, increased nucleocytoplasmic ratio, and decreased extracellular space. The pathophysiological basis of tumor invasiveness is complex and involves multiple mechanisms; therefore, the precise relationship of pathological findings with radiomics features, especially higher-order features, remains to be fully elucidated (29).

Few studies have investigated the radiomics features of TETs based on CT images (16). We have created a highly prognostic radiomics model that was validated in two independent datasets of TET patients. The prediction ability of the radiomics model was greatly improved, compared with that of 


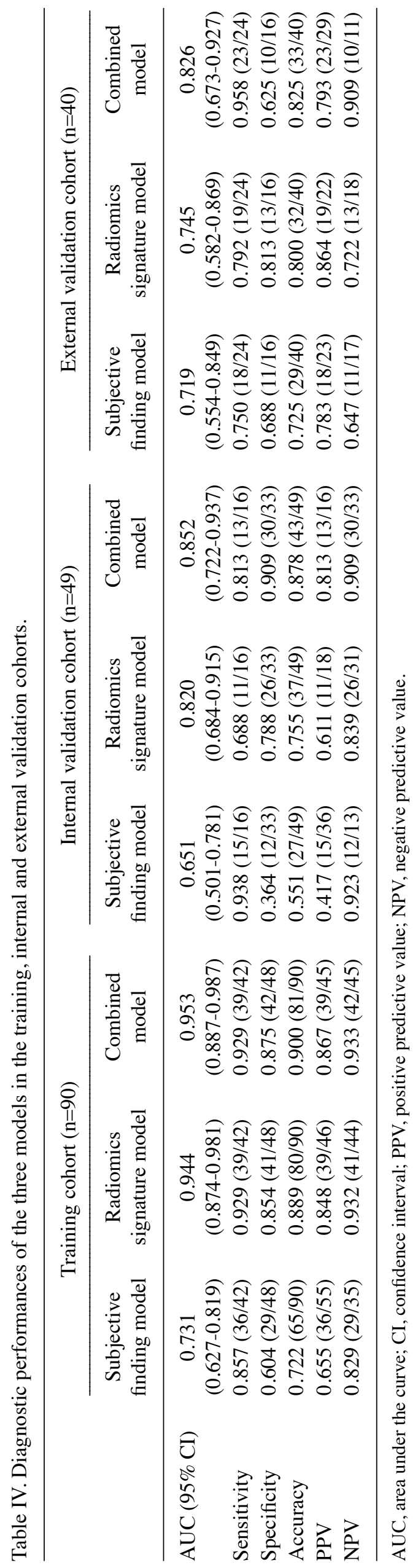

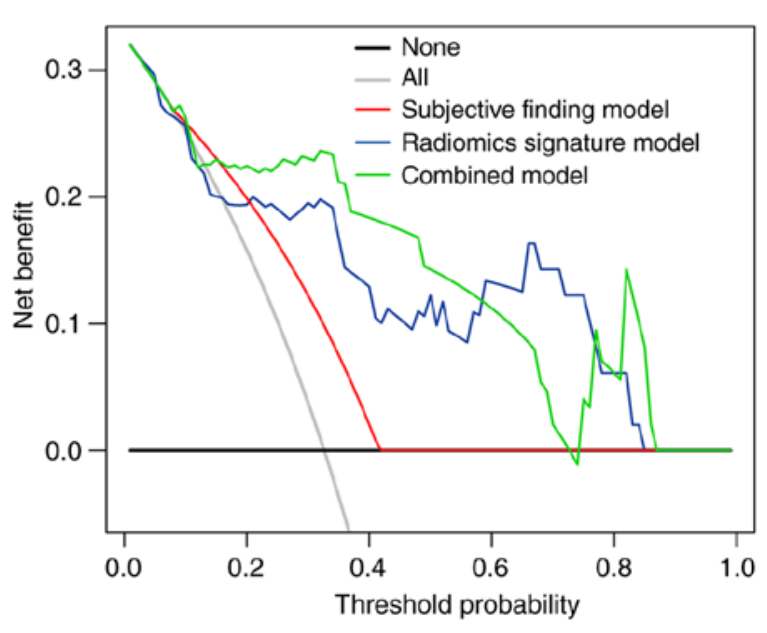

Figure 6. Decision-curve analysis. The net benefit of each model is plotted on the $y$-axis. The $x$-axis indicates the threshold probability, which is when the expected benefit of the receiving the treatment is the same as that of avoiding the treatment. The subjective finding, radiomics signature, and combined models are indicated by the red, blue, and green lines, respectively. The gray and black lines indicate the assumptions that all or no patients have high-risk TETs, respectively. The combined model provides the highest net benefit (threshold probability, 0.01-0.87). TETs, thymic epithelial tumors.

the subjective finding model, and significant differences were found between the AUCs of the two models in the training, and internal and external validation cohorts (DeLong test: $\mathrm{P}<0.0001$, $\mathrm{P}=0.0187, \mathrm{P}=0.0169$, respectively). As the information of subjective findings may only take into consideration certain aspects of TETs, a better diagnostic performance can be achieved by integrating subjective findings with radiomics features to create a radiomics model. We used venous-phase images rather than non-enhanced or arterial-phase images because venous-phase images enable improved lesion visualization and accurate segmentation of anterior mediastinal neoplasms, which are surrounded by mediastinal fat tissue, large vessels, pleura, and lung parenchyma. Moreover, venous-phase images have previously been used to reveal enhancement heterogeneity for the radiomics analysis of soft-tissue neoplasms, such as gastric cancer, renal tumor, and hepatocellular cancer (30-32). We selected 3D radiomics features over $2 \mathrm{D}$ features because the former provide comprehensive information and improve the accuracy of radiomics-based predictions (33). All extracted features with ICC values $>0.75$ were analyzed using LASSO logistic regression in our study.

The present study has certain limitations. First of all, the retrospective study design was associated with selection bias, and the sample size was relatively small, although datasets from two hospitals were collected independently. A larger prospective, multicenter study is required to validate our preliminary results. Secondly, the use of four different CT scanners may have influenced the evaluation of some CT findings owing to partial volume effects. However, all patient CT images were reconstructed using a slice thickness $\leq 3.0 \mathrm{~mm}$, and multiplanar reconstruction including coronal and sagittal planes was applied. Moreover, there were no significant differences in the stratification analyses of CT devices and CT slice thicknesses. Thirdly, lesions were manually segmented slice-by-slice and semi-automatically delineated. Further research and the development of advanced, automated segmentation techniques are needed for 
more widespread clinical implementation of radiomics-based prediction models in the future. Finally, radiomics signatures were extracted from enhanced CT images in our study since thoracic enhanced CT scans are routinely performed for patients with clinical suspected mediastinal masses. A radiomics model combining conventional CT images with more detailed and informative imaging biomarkers as well as positron emission tomography/computed tomography (PET/CT) parameters could substantially enhance the predictive value in the risk status of TETs. In the future, we will recruit more patients to increase the sample size and include more patients with PET/CT data to update our results.

In conclusion, our results suggest that enhanced CT-based radiomics signature is a noninvasive, reliable, and reproducible imaging marker that may help to assess the pathological risk of TETs. Our combined model, which included both the radiomics signature and a subjective finding, may greatly facilitate the preoperative identification of low-risk and high-risk TETs.

\section{Acknowledgements}

Not applicable.

\section{Funding}

No funding was received.

\section{Availability of data and materials}

All data generated or analyzed during the present study are included in this published article or are available from the corresponding author on reasonable request.

\section{Authors' contributions}

Study concept and design were carried out by $\mathrm{XC}, \mathrm{ZL}, \mathrm{BF}$ and WL. Literature research was collected by CL and YC. Clinical studies were conducted by XC, XD, ZL, and CZ. Data and statistical analyses were performed by $\mathrm{CL}$ and $\mathrm{XD}$. XC, WL and $\mathrm{BF}$ guarantee the integrity of the entire research study. All authors read and approved the manuscript and agree to be accountable for all aspects of the research in ensuring that the accuracy or integrity of any part of the work are appropriately investigated and resolved.

\section{Ethics approval and consent to participate}

The study protocol was approved by the Institutional Review Boards of Jiangmen Central Hospital (Jiangmen, Guangdong, China) and The Fifth Affiliated Hospital of Sun Yat-sen University (Zhuhai, Guangdong, China). Informed consent was waived as the study had a retrospective design.

\section{Patient consent for publication}

Not applicable.

\section{Competing interests}

The authors declare that they have no competing interests.

\section{References}

1. Marom EM: Advances in thymoma imaging. $\mathrm{J}$ Thorac Imaging 28: 69-83, 2013.

2. Fujii Y: Published guidelines for management of thymoma. Thorac Surg Clin 21: 125-129, 2011.

3. Marx A, Ströbel P, Badve SS, Chalabreysse L, Chan JK, Chen G, de Leval L, Detterbeck F, Girard N, Huang J, et al: ITMIG consensus statement on the use of the WHO histological classification of thymoma and thymic carcinoma: Refined definitions, histological criteria, and reporting. J Thorac Oncol 9: 596-611, 2014.

4. Marx A, Chan JK, Coindre JM, Detterbeck F, Girard N, Harris NL, Jaffe ES, Kurrer MO, Marom EM, Moreira AL, et al: The 2015 World Health Organization classification of tumors of the thymus: Continuity and changes. J Thorac Oncol 10: 1383-1395, 2015.

5. Giaccone G, Wilmink H, Paul MA and van der Valk P: Systemic treatment of malignant thymoma. Am J Clin Oncol 29: 336-344, 2006.

6. Okumura M, Miyoshi S, Fujii Y, Takeuchi Y, Shiono H, Inoue M, Fukuhara K, Kadota Y, Tateyama H, Eimoto T and Matsuda H: Clinical and functional significance of WHO classification on human thymic epithelial neoplasms: A study of 146 consecutive tumors. Am J Surg Pathol 25: 103-110, 2001.

7. Falkson CB, Bezjak A, Darling G, Gregg R, Malthaner R, Maziak DE, Yu E, Smith CA, McNair S, Ung YC, et al: The management of thymoma: A systematic review and practice guideline. J Thorac Oncol 4: 911-919, 2009.

8. Jeong YJ, Lee KS, Kim J, Shim YM, Han J and Kwon OJ: Does CT of thymic epithelial tumors enable us to differentiate histologic subtypes and predict prognosis? AJR Am J Roentgenol 183: 283-289, 2004.

9. Marom EM, Milito MA, Moran CA, Liu P, Correa AM, Kim ES, Komaki R, Erasmus JJ, Hofstetter WL, Rice DC and Swisher SG: Computed tomography findings predicting invasiveness of thymoma. J Thorac Oncol 6: 1274-1281, 2011.

10. Liu GB, Qu YJ, Liao MY, Hu HJ, Yang GF and Zhou SJ: Relationship between computed tomography manifestations of thymic epithelial tumors and the WHO pathological classification. Asian Pac J Cancer Prev 13: 5581-5585, 2012.

11. Lambin P, Rios-Velazquez E, Leijenaar R, Carvalho S, van Stiphout RG, Granton P, Zegers CM, Gillies R, Boellard R, Dekker A and Aerts HJ: Radiomics: Extracting more information from medical images using advanced feature analysis. Eur $\mathbf{J}$ Cancer 48: 441-446, 2012.

12. Kumar V, Gu Y, Basu S, Berglund A, Eschrich SA, Schabath MB, Forster K, Aerts HJ, Dekker A, Fenstermacher D, et al: Radiomics: The process and the challenges. Magn Reson Imaging 30: 1234-1248, 2012.

13. Aerts HJ, Velazquez ER, Leijenaar RT, Parmar C, Grossmann P, Carvalho S, Bussink J, Monshouwer R, Haibe-Kains B, Rietveld $\mathrm{D}$, et al: Decoding tumour phenotype by noninvasive imaging using a quantitative radiomics approach. Nat Commun 5: 4006, 2014.

14. Braman NM, Etesami M, Prasanna P, Dubchuk C, Gilmore H, Tiwari P, Plecha D and Madabhushi A: Intratumoral and peritumoral radiomics for the pretreatment prediction of pathological complete response to neoadjuvant chemotherapy based on breast DCE-MRI. Breast Cancer Res 19: 57, 2017.

15. Kuo MD and Jamshidi N: Behind the numbers: Decoding molecular phenotypes with radio-genomics-Guiding principles and technical considerations. Radiology 270: 320-325, 2014.

16. Iannarelli A, Sacconi B, Tomei F, Anile M, Longo F, Bezzi M, Napoli A, Saba L, Anzidei M, D'Ovidio G, et al: Analysis of CT features and quantitative texture analysis in patients with thymic tumors: Correlation with grading and staging. Radiol Med 123: 345-350, 2018.

17. Priola AM, Priola SM, Di Franco M, Cataldi A, Durando S and Fava C: Computed tomography and thymoma: Distinctive findings in invasive and noninvasive thymoma and predictive features of recurrence. Radiol Med 115: 1-21, 2010 (In English, Italian).

18. Carter BW, Benveniste MF, Madan R, Godoy MC, Groot PM, Truong MT, Rosado-de-Christenson ML and Marom EM: IASLC/ITMIG staging system and lymph node map for thymic epithelial neoplasms. Radiographics 37: 758-776, 2017.

19. Landis JR and Koch GG: The measurement of observer agreement for categorical data. Biometrics 33: 159-174, 1977. 
20. Shrout PE and Fleiss JL: Intraclass correlations: Uses in assessing rater reliability. Psychol Bull 86: 420-428, 1979.

21. Vickers AJ, Cronin AM, Elkin EB and Gonen M: Extensions to decision curve analysis, a novel method for evaluating diagnostic tests, prediction models and molecular markers. BMC Med Inform Decis Mak 8: 53, 2008.

22. Tomiyama N, Johkoh T, Mihara N, Honda O, Kozuka T, Koyama M, Hamada S, Okumura M, Ohta M, Eimoto T, et al: Using The World Health Organization classification of thymic epithelial neoplasms to describe CT findings. AJR Am J Roentgenol 179: 881-886, 2002.

23. Sadohara J, Fujimoto K, Müller NL, Kato S, Takamori S, Ohkuma K, Terasaki H and Hayabuchi N: Thymic epithelial tumors: Comparison of CT and MR imaging findings of low-risk thymomas, high-risk thymomas, and thymic carcinomas. Eur J Radiol 60: 70-79, 2006

24. Tomiyama N, Müller NL, Ellis SJ, Cleverley JR, Okumura M, Miyoshi S, Kusumoto M, Johkoh T, Yoshida S, Mihara N, et al: Invasive and non-invasive thymoma: Distinctive CT features. J Comput Assist Tomogr 25: 388-393, 2001.

25. Choe J, Lee SM, Lim S, Choi SH, Kim N, Do KH and Seo JB: Doubling time of thymic epithelial tumours on CT: Correlation with histological subtype. Eur Radiol 27: 4030-4036, 2017.

26. Henschke CI, Lee IJ, Wu N, Farooqi A, Khan A, Yankelevitz D and Altorki NK: CT screening for lung cancer: Prevalence and incidence of mediastinal masses. Radiology 239: 586-589, 2006.

27. Detterbeck FC: Clinical value of the WHO classification system of thymoma. Ann Thorac Surg 81: 2328-2334, 2006.
28. Qu YJ, Liu GB, Shi HS, Liao MY, Yang GF and Tian ZX: Preoperative CT findings of thymoma are correlated with postoperative Masaoka clinical stage. Acad Radiol 20: 66-72, 2013.

29. Lambin P, Leijenaar RTH, Deist TM, Peerlings J, de Jong EEC, van Timmeren J, Sanduleanu S, Larue RTHM, Even AJG, Jochems A, et al: Radiomics: The bridge between medical imaging and personalized medicine. Nat Rev Clin Oncol 14: 749-762, 2017.

30. Ma Z, Fang M, Huang Y, He L, Chen X, Liang C, Huang X, Cheng Z, Dong D, Liang C, et al: CT-based radiomics signature for differentiating Borrmann type IV gastric cancer from primary gastric lymphoma. Eur J Radiol 91: 142-147, 2017.

31. Kocak B, Ates E, Durmaz ES, Ulusan MB and Kilickesmez O: Influence of segmentation margin on machine learning-based high-dimensional quantitative CT texture analysis: A reproducibility study on renal clear cell carcinomas. Eur Radiol 12: 4765-4775, 2019.

32. Ma X, Wei J, Gu D, Zhu Y, Feng B, Liang M, Wang S, Zhao X and Tian J: Preoperative radiomics nomogram for microvascular invasion prediction in hepatocellular carcinoma using contrast-enhanced CT. Eur Radiol 29: 3595-3605, 2019.

33. Shen C, Liu Z, Guan M, Song J, Lian Y, Wang S, Tang Z, Dong D, Kong L, Wang M, et al: 2D and 3D CT radiomics features prognostic performance comparison in non-small cell lung cancer. Transl Oncol 10: 886-894, 2017.

This work is licensed under a Creative Commons Attribution-NonCommercial-NoDerivatives 4.0 International (CC BY-NC-ND 4.0) License. 